\title{
Sex differences in gene expression and proliferation are dependent on the epigenetic modifier HP1 $\gamma$
}

Pui-Pik Law ${ }^{1,2,4} \dagger$, Ping-Kei Chan ${ }^{1,2} \dagger$, Kirsten McEwen ${ }^{3}$, Huihan Zhi ${ }^{1}$, Bing Liang ${ }^{1,2}$, Chie Naruse ${ }^{6}$, Masahide Asano ${ }^{6}$, Kian-Cheng Tan-Un ${ }^{5}$, Godfrey Chi-Fung Chan ${ }^{4}$, Richard Festenstein ${ }^{1,2 *}$

${ }^{1}$ Gene Control Mechanisms and Disease Group, Department of Medicine, Imperial College London, London, United Kingdom

${ }^{2}$ Epigenetics Section, MRC London Institute of Medical Sciences, Faculty of Medicine, Imperial College London, London, United Kingdom

${ }^{3}$ Department of Life Sciences, Faculty of Natural Sciences, Imperial College London, London, United Kingdom

${ }^{4}$ Department of Pediatrics \& Adolescent Medicine, LKS Faculty of Medicine, The University of Hong Kong, Hong Kong

${ }^{5}$ School of Biological Sciences, The University of Hong Kong, Hong Kong

${ }^{6}$ Institute of Laboratory Animals, Graduate School of Medicine, Kyoto University, Kyoto, Japan

†These authors contributed equally to this work.

*To whom correspondence should be addressed. E-mail: r.festenstein@imperial.ac.uk

\section{Summary}

Sex differences in growth rate in very early embryos have been recognized in a variety of mammals and attributed to sex-chromosome complement effects as they occur before overt sexual differentiation. We previously found that sex-chromosome complement, rather than sex hormones regulates heterochromatin-mediated silencing of a transgene and autosomal gene expression in mice. Here, sex dimorphism in proliferation was investigated. We confirm that male embryonic fibroblasts proliferate faster than female fibroblasts and show that this proliferation advantage is completely dependent upon heterochromatin protein 1 gamma (HP1 $\gamma)$. To determine whether this sex-regulatory effect of HP1 $\gamma$ was a more general phenomenon, we performed RNA sequencing on MEFs derived from males and females, with or without HP1 $\gamma$. Strikingly, HP1 $\gamma$ was found to be crucial for regulating nearly all sexually dimorphic autosomal gene expression because deletion of the HP1 $\gamma$ gene in males abolished sex differences in autosomal gene expression. The identification of a key epigenetic modifier as central 
in defining gene expression differences between males and females has important implications for understanding physiological sex differences and sex bias in disease.

\section{Introduction}

Sex dimorphism in physiology as well as gene expression pattern has been observed in many animal species including mice ${ }^{1-6}$ and humans ${ }^{7-10}$ during early development. For example, sex dimorphism in growth rate of mammalian embryos before gonadal sex differentiation has been shown by a number of previous studies, where males were found to be developmentally more advanced relative to females ${ }^{2-4}$. These differences are attributed to the difference in sex chromosome complement which are the only factors that differ between male (XY) and female (XX) zygotes and are thought to set up life-long sex differences. Indeed, using the 'Four Core Genotypes' mouse model, a number of studies have shown the crucial role of sex chromosome complement, independent of hormones in exerting divergent effects over metabolic and behavioural phenotypes in adults ${ }^{11-13}$. Further understanding of how sex dimorphism is regulated during early development is therefore important as sex chromosomes may differentially prime the genome and impact on developmental programming and other sexual differences (including disease susceptibility) later in life ${ }^{14,15}$. However, little is known about the molecular mechanisms underlying these sexual differences.

Heterochromatin protein 1 (HP1) is a non-histone chromosomal protein that was initially discovered in Drosophila to be enriched at heterochromatic regions ${ }^{16}$. HP1 is highly conserved through evolution and its homologues are found in almost all eukaryotes except Saccharomyces cerevisiae ${ }^{17}$. In mammals, the three HP1 isoforms alpha (HP1 $\alpha$ ), beta (HP1 $\beta)$ and gamma (HP1 $\gamma$ ) display a high degree of structural and biochemical similarity ${ }^{17}$ but appear to have distinct nuclear localisation ${ }^{18-21}$, expression profiles $^{22}$, non-redundant functions ${ }^{23-27}$ and isoform-specific binding partners ${ }^{28}$. Among the three HP1 isoforms in mammals, HP1 $\gamma$ is distinguished by its unique subnuclear localisation pattern in both euchromatic and heterochromatic regions in interphase nuclei ${ }^{18,21,29,30}$.

HP1 has been regarded as a silencing protein with its interaction with the histone mark H3K9 methylation that associated predominately with silent heterochromatin ${ }^{31-33}$ as well as its ability to multimerise to interact with the H3K9 methyltransferase ${ }^{34}$. However, evidence accumulated in the last decade has implicated HP1 in a wide range of nuclear functions ${ }^{35}$. Of particular interest, is the implication that HP1 $\gamma$ participates 
in gene activation: (1) It has been shown that depletion of HP1 $\gamma$ in mammalian cells can result in down-regulation of gene expression ${ }^{36,37}$, (2) HP1 $\gamma$ has been shown to associate with transcriptionally active genes ${ }^{36,38,39}$ and (3) interact with the RNA polymerase II ${ }^{38,39}$ and mediator components ${ }^{36}$. Yet, the exact nature and mechanisms whereby HP1 $\gamma$ contributes positively to gene expression is unclear.

Previously, it has been shown that depletion of a Drosophila HP1 homologue, HP1a, resulted in male-biased lethality ${ }^{40}$. In mice, HP1 $\gamma$ deletion resulted in growth retardation ${ }^{41}$, however, in that study both placental insufficiency and impaired proliferation were implicated as potential causes. Although defects in germ cell development have been identified in both sexes ${ }^{42,43}$ in HP1 $\gamma$ deficient mice, another study showed that one female with a hypomorphic mutation on both alleles of HP1 $\gamma$ had normal ovaries ${ }^{26}$. These findings, together with our previous finding implicating HP1 in the regulation of sex-dimorphic autosomal gene expression in murine thymic cells ${ }^{44}$ raised the question as to whether HP1 was also contributing to sex dimorphism in early development of mice.

We directly addressed here whether HP1 $\gamma$ is involved in regulation of sex difference in early development. Using an HP1 $\gamma$ knockout mouse model, we found that the proliferation advantage of the primary embryonic fibroblasts derived from male E13.5 embryos is dependent upon the epigenetic modifier HP1 $\gamma$. Strikingly, analysis of MEF's transcriptome revealed that HP1 $\gamma$ is crucial for maintaining nearly all sex differences in autosomal gene expression by regulating the expression level of these genes in males.

\section{Results}

Sex dimorphism in cellular proliferation rate of primary mouse embryonic fibroblasts is dependent on HP1y

In line with previous studies ${ }^{2-4}$, we observed a higher proliferation rate in male mouse embryonic fibroblasts (MEFs) derived from embryonic day 13.5 (E13.5) embryos than in female MEFs (Figure 1A). To investigate this sex difference further, we analysed the genome-wide transcriptional profile of these cells by RNA-sequencing (RNA-seq). Using Gene Set Enrichment Analysis (GSEA), we found a significant difference in the expression of a set of cell cycle related genes between males and females (Figure 1C and Figure S1A), consistent with the observed difference in cell proliferation rate.

Strikingly, upon removal of HP1 $\gamma$ (Figure 2), the normally higher proliferation rate of 
male wild type (WT) MEFs was reduced to a comparable level to that observed in WT females while the proliferation rate of female MEFs was largely unaffected (Figure 1B). GSEA of the transcriptomic data of these cells also showed a significant change in a set of cell cycle related genes in males but not in females upon HP1 $\gamma$ knockout (KO) (Figure 1D, 1E and Figure S1B, S1C). The difference in proliferation in WT male and female MEFs does not appear to be due to the difference in HP1 $\gamma$ level as a similar level of HP1 $\gamma$ gene expression was found in both WT male and female MEFs (Figure S3). Thus, depletion of HP1 $\gamma$ specifically affects proliferation rate of male MEFs and hence equalized the proliferation rate between the sexes. Thus, these data suggest that HP1 $\gamma$ is required for maintaining the sex difference in growth rate.

\section{HP1 $y$ regulates sexual dimorphic expression of autosomal genes}

The observed differences in response to HP1 $\gamma$ deficiency in males and females prompted us to ask how broad HP1 $\gamma$ 's role is in regulating sex-specific gene expression and hence contributing to the maintenance of sex differences. To address this question, sexually dimorphic genes (defined as genes showing $\geq 1$.5-fold difference between WT males and females (P-value $<0.05)$ ) were identified from the transcriptomic data derived from the MEFs (Figure 2A and Table S2, S3) and tested for their sensitivity to HP1 $\gamma$ deficiency in both males and females. As well as genes encoded by the sex chromosomes, autosomal genes also showed dimorphic expression when WT males and females are compared (Figure 2A). These genes fall into two categories (1) 114 'malelower genes' that express at a lower level in WT males compared to females (Figure 2B(I)) and (2) 62 'male-higher genes' that express at a higher level in WT males than females (Figure 2C(I))).

Strikingly, in males, the 'male-lower genes' were nearly all upregulated in the absence of HP1 $\gamma$ (Figure 2B(II)). In contrast, most of these genes were not changed in females upon HP1 $\gamma$ KO (Figure 2B(III)). These results suggest that, in the WT condition, HP1 $\gamma$ has an important role in repressing these genes in males and maintains sexually dimorphic gene expression. Indeed, direct comparison of the male HP1 $\gamma$ KO MEFs with the WT female MEFs revealed a marked loss of sex difference in the expression of these genes (Figure 2B(IV)). Almost all of the 62 'male-higher genes' (Figure 2C(I)) were downregulated when HP1 $\gamma$ was knocked out (Figure 2C(II)), suggesting that in males most of these genes require HP1 $\gamma$ to keep them at a higher level than that seen in females. Comparison of the male HP1 $\gamma$ KO MEFs with the WT female MEFs revealed that HP1 $\gamma$ was required for the sex-difference in gene expression of these genes (Figure 2C(IV)). Similar to the 'male-lower genes', a minimal effect was observed on the 'male-higher 
genes' in females upon HP1 $\gamma$ KO (Figure 2C(III)). The changes in expression of a subset of the sexually dimorphic genes were confirmed by quantitative real-time PCR (qRT-PCR) (Figure S4). To test whether the observed dysregulation of these sexually dimorphic genes in response to HP1 $\gamma \mathrm{KO}$ was an artifact of the analysis of a small group of genes (i.e. 176 genes out of 23420 genes), 176 randomly selected non-sexually dimorphic genes were also examined for their sensitivity to HP1 $\gamma$ deficiency (Figure $\mathrm{S} 5 \mathrm{~A}$ ). In the absence of HP1 $\gamma$, these genes were largely unaffected in both sexes (Figure S5B and S5C). These genes were also expressed at a similar level in HP1 $\gamma$ KO males compared to WT females (Figure S5D). Overall, these results implicate HP1 $\gamma$ in regulating the sexually dimorphic gene expression in MEFs and show that the effect is greater in male MEFs than female, suggesting an essential role for HP1 $\gamma$ in establishing or maintaining the difference in autosomal gene expression between the sexes.

\section{HP1 $y$ deficiency leads to sex-biased dysregulation of a range of biological processes that are known to differ between the sexes}

Our results revealed a male-specific defect in cell proliferation rate as well as dysregulation of sexually dimorphic gene expression in response to HP1 $\gamma$ deficiency. To investigate whether HP1 $\gamma$ may also affect other cellular processes in a sex-biased manner, genes that were specifically affected in males or females in response to HP1 $\gamma$ deficiency were identified (Figure 3A-3C) and the functional relevance of these genes was revealed using Gene Ontology (GO) analysis (Figure 4 and Tables S4 and S5). Consistent with previous studies, both up- and down-regulated genes were identified upon HP1 $\gamma$ KO in our system, implicating HP1 $\gamma$ in both the activation and repression of gene expression (Figure $3 \mathrm{~A}$ and $3 \mathrm{~B})^{36,37}$. A dramatic sexually-dimorphic transcriptional defect in response to HP1 $\gamma$ KO was observed (Figure 3A and 3B). 3827 genes were specifically dysregulated in males but only 720 genes in females by at least 1.5-fold $(\mathrm{P}<0.05)$ (Figure 3C). 466 genes were dysregulated in both males and females (Figure 3C). This sex-biased response to HP1 $\gamma$ deficiency is reminiscent of the previously reported difference between males and females upon depletion of HP1a in Drosophila $^{40}$ suggesting evolutionary conservation. Gene Ontology (GO) analysis of genes regulated by HP1 $\gamma$ revealed a larger number of biological processes associated with genes affected in males (47) compared to females (20) (Figure 4 and Tables S4 and S5). These include several biological processes reported to differ between the sexes such as progression of cell cycle ${ }^{2-4}$ as well as immune system related functions ${ }^{45}$ (Figure 4 and Tables S4 and S5). Interestingly, 'glucose homeostasis' which is also known to differ between the sexes ${ }^{46,47}$ is found in the female affected genes but not the male 
affected genes. Notably, as well as potentially regulating sex-bias in embryonic growth, cell-cycle related differences are known to be important for sex-dimorphism in cancer susceptibility ${ }^{48}$, moreover, response to infection and susceptibility to autoimmunity is well known to differ between the sexes.

\section{Discussion}

The results obtained in the current study suggest a male-specific role for HP1 $\gamma$ in regulating sexually dimorphic gene expression which in turn contributes to sexual differences. This is perhaps a surprising finding because HP1 $\gamma$ was found to be expressed at a comparable level in males and females (Figure S3). Interestingly, a study in Drosophila revealed male sex-bias in the effect on gene regulation upon depletion of the HP1 homolog HP1a ${ }^{40}$ although that study did not examine the effect on sex dimorphic gene expression. Taken together with our results these findings might suggest evolutionary conservation of sex-specific effects of HP1 on gene regulation.

How the male-specific regulation of the sexually dimorphic genes is mediated by HP1 $\gamma$ remains to be further studied. In this study, sex-specific effects of HP1 $\gamma$ were studied in mouse embryonic fibroblasts derived from embryos at E13.5 whose fetal Leydig cells have just started to produce androgen ${ }^{51}$. It is therefore possible that the very low levels of testosterone found in these male embryos might contribute to the targeting of HP1 $\gamma$ in a sex-specific manner. However, regulation of pituitary growth hormone secretion by gonadal hormones before puberty in mice was shown to be minimal ${ }^{52}$. Thus, sexual difference observed here is likely to be regulated by sex chromosome complement rather than just sex hormones ${ }^{53}$. It is tempting to speculate that HP1 $\gamma$ may interact directly or indirectly with one or more factors contributed by the sex chromosome complement to give rise to the sex-specific effect observed.

The $\mathrm{Y}$ chromosome which is uniquely present in males may encode factors that contribute to HP1 $\gamma$ 's male-specific regulation of the 'male-higher' genes in males. It has been shown previously that HP1 $\gamma$ associates with the protein encoded by the Sry gene, which is crucial for male sexual development ${ }^{54}$ through indirect interaction with KAP1 and KRAB-O protein ${ }^{55}$. However, most of the Y chromosome genes, including Sry, are largely repressed in MEFs with only four of them showing pronounced expression in our transcriptomic data. Two genes were of particular interest, Uty and Kdm5d which encode H3K27me3 and H3K4me3 demethylases respectively ${ }^{56,57}$ (Table S7). 
Although dosage difference of $\mathrm{X}$ chromosomes between males and females is compensated by $\mathrm{X}$ chromosome inactivation in female cells ${ }^{58}$, a number of $\mathrm{X}$-linked genes were found to escape $\mathrm{X}$-inactivation ${ }^{59}$. Indeed, we observed in our transcriptomic data female-biased expression of several known X-escapees ${ }^{59}$ (Kdm5c, Eif2s3x and $\mathrm{Kdm6a}$ ) as well as some other $\mathrm{X}$-linked genes which have not been previously reported as escaping X-inactivation (5530601H04Rik, 2900056M20Rik and Ppef1) (Table S7). To contribute to the male-specific regulation of the 'male-higher' genes, the proteins encoded by these X-escapees may compete with HP1 $\gamma$ for binding to these genes in females to protect them from the activating effect of HP1 $\gamma$.

Interestingly, when looking at the list of sex chromosome genes that are differentially expressed in males and females, we found two X-linked genes (Ppef1 and 2900056M20Rik) which show female-biased expression and were upregulated in response to HP1 $\gamma$ knockout in males. Importantly, upon removal of HP1 $\gamma$ in males, the difference in expression level of these genes between males (KO) and females (WT) became insignificant (Table S8). Ppef1 encodes a serine/threonine-protein phosphatase $^{60}$. Although this protein has not been shown to regulate gene expression directly, being a protein phosphatase, it is possible that it regulates gene expression indirectly by activating or deactivating other protein factors. 2900056M20Rik, also known as Kantr (Kdm5c adjacent non-coding transcript) which encodes a long noncoding RNA. It has been shown previously that deletion of 2900056M20Rik results in an increase in expression of sets of genes involved in neuronal development and various cell signaling pathways and in murine embryonic and adult brain ${ }^{61}$. It is possible that the higher expression level of these genes in females is responsible for keeping the higher expression of these 'male-lower' genes in females compared to males. Removal of HP1 $\gamma$ may upregulate expression of Ppef1 and 2900056M20Rik in males and lead to upregulation of the 'male-lower' genes in males and normalization of sex difference.

Finally, it has been hypothesised that the inactive X-chromosome in females that is decorated with H3K9me $3^{62}$ might be acting as a heterochromatic 'sink' ${ }^{63}$ thereby sequestering HP1 $\gamma$ away from the euchromatic regions. Such an effect would operate in females effectively reducing the availability of HP1

In summary, we have identified the epigenetic modifier HP1 $\gamma$ as an essential component in the regulation of autosomal gene expression differences between the sexes which is likely to be important for understanding sex dimorphisms in physiology and disease. 


\section{References:}

1. Lowe, R., Gemma, C., Rakyan, V.K. \& Holland, M.L. Sexually dimorphic gene expression emerges with embryonic genome activation and is dynamic throughout development. BMC Genomics 16, 295 (2015).

2. Burgoyne, P.S. et al. The genetic basis of XX-XY differences present before gonadal sex differentiation in the mouse. Philos Trans $R$ Soc Lond B Biol Sci 350, 253-60 discussion 260-1 (1995).

3. Burgoyne, P.S. A Y-chromosomal effect on blastocyst cell number in mice. Development 117, 341-5 (1993).

4. Thornhill, A.R. \& Burgoyne, P.S. A paternally imprinted X chromosome retards the development of the early mouse embryo. Development 118, 171-4 (1993).

5. Peippo, J. \& Bredbacka, P. Sex-related growth rate differences in mouse preimplantation embryos in vivo and in vitro. Mol Reprod Dev 40, 56-61 (1995).

6. Valdivia, R.P., Kunieda, T., Azuma, S. \& Toyoda, Y. PCR sexing and developmental rate differences in preimplantation mouse embryos fertilized and cultured in vitro. Mol Reprod Dev 35, 121-6 (1993).

7. Menezo, Y.J., Chouteau, J., Torello, J., Girard, A. \& Veiga, A. Birth weight and sex ratio after transfer at the blastocyst stage in humans. Fertil Steril 72, 221-4 (1999).

8. $\quad$ Pergament, E., Fiddler, M., Cho, N., Johnson, D. \& Holmgren, W.J. Sexual differentiation and preimplantation cell growth. Hum Reprod 9, 1730-2 (1994).

9. Ray, P.F., Conaghan, J., Winston, R.M. \& Handyside, A.H. Increased number of cells and metabolic activity in male human preimplantation embryos following in vitro fertilization. J Reprod Fertil 104, 165-71 (1995).

10. Ronen, D. \& Benvenisty, N. Sex-dependent gene expression in human pluripotent stem cells. Cell Rep 8, 923-32 (2014).

11. Chen, $\mathrm{X}$. et al. The number of $\mathrm{x}$ chromosomes causes sex differences in adiposity in mice. PLoS Genet 8, e1002709 (2012).

12. Chen, X., McClusky, R., Itoh, Y., Reue, K. \& Arnold, A.P. X and Y chromosome complement influence adiposity and metabolism in mice. Endocrinology 154, 1092-104 (2013).

13. Kuljis, D.A. et al. Gonadal- and sex-chromosome-dependent sex differences in the circadian system. Endocrinology 154, 1501-12 (2013).

14. Aiken, C.E. \& Ozanne, S.E. Sex differences in developmental programming 
models. Reproduction 145, R1-13 (2013).

15. Donjacour, A., Liu, X., Lin, W., Simbulan, R. \& Rinaudo, P.F. In vitro fertilization affects growth and glucose metabolism in a sex-specific manner in an outbred mouse model. Biol Reprod 90, 80 (2014).

16. James, T.C. \& Elgin, S.C. Identification of a nonhistone chromosomal protein associated with heterochromatin in Drosophila melanogaster and its gene. $\mathrm{Mol}$ Cell Biol 6, 3862-72 (1986).

17. Jones, D.O., Cowell, I.G. \& Singh, P.B. Mammalian chromodomain proteins: their role in genome organisation and expression. Bioessays 22, 124-37 (2000).

18. Minc, E., Allory, Y., Worman, H.J., Courvalin, J.C. \& Buendia, B. Localization and phosphorylation of HP1 proteins during the cell cycle in mammalian cells. Chromosoma 108, 220-34 (1999).

19. Kourmouli, N., Sun, Y.M., van der Sar, S., Singh, P.B. \& Brown, J.P. Epigenetic regulation of mammalian pericentric heterochromatin in vivo by HP1. Biochem Biophys Res Commun 337, 901-7 (2005).

20. Nielsen, A.L. et al. Heterochromatin formation in mammalian cells: interaction between histones and HP1 proteins. Mol Cell 7, 729-39 (2001).

21. Horsley, D., Hutchings, A., Butcher, G.W. \& Singh, P.B. M32, a murine homologue of Drosophila heterochromatin protein 1 (HP1), localises to euchromatin within interphase nuclei and is largely excluded from constitutive heterochromatin. Cytogenet Cell Genet 73, 308-11 (1996).

22. Kwon, S.H. \& Workman, J.L. The changing faces of HP1: From heterochromatin formation and gene silencing to euchromatic gene expression: HP1 acts as a positive regulator of transcription. Bioessays 33, 280-9 (2011).

23. Dialynas, G.K. et al. Plasticity of HP1 proteins in mammalian cells. J Cell Sci 120, 3415-24 (2007).

24. Lee, D.H. et al. DNA microarray profiling of genes differentially regulated by three heterochromatin protein 1 (HP1) homologs in Drosophila. Biochem Biophys Res Commun 434, 820-8 (2013).

25. Ha, N. et al. HP-1gamma Controls High-Affinity Antibody Response to TDependent Antigens. Front Immunol 5, 271 (2014).

26. Brown, J.P. et al. HP1gamma function is required for male germ cell survival and spermatogenesis. Epigenetics Chromatin 3, 9 (2010).

27. Aucott, R. et al. HP1-beta is required for development of the cerebral neocortex and neuromuscular junctions. J Cell Biol 183, 597-606 (2008).

28. Kwon, S.H. \& Workman, J.L. The heterochromatin protein 1 (HP1) family: put away a bias toward HP1. Mol Cells 26, 217-27 (2008). 
29. Minc, E., Courvalin, J.C. \& Buendia, B. HP1gamma associates with euchromatin and heterochromatin in mammalian nuclei and chromosomes. Cytogenet Cell Genet 90, 279-84 (2000).

30. Nielsen, A.L. et al. Interaction with members of the heterochromatin protein 1 (HP1) family and histone deacetylation are differentially involved in transcriptional silencing by members of the TIF1 family. EMBO J 18, 6385-95 (1999).

31. Lachner, M., O'Carroll, D., Rea, S., Mechtler, K. \& Jenuwein, T. Methylation of histone H3 lysine 9 creates a binding site for HP1 proteins. Nature $\mathbf{4 1 0}$, 116-20 (2001).

32. Bannister, A.J. et al. Selective recognition of methylated lysine 9 on histone H3 by the HP1 chromo domain. Nature 410, 120-4 (2001).

33. Jacobs, S.A. \& Khorasanizadeh, S. Structure of HP1 chromodomain bound to a lysine 9-methylated histone H3 tail. Science 295, 2080-3 (2002).

34. Yamamoto, K. \& Sonoda, M. Self-interaction of heterochromatin protein 1 is required for direct binding to histone methyltransferase, SUV39H1. Biochem Biophys Res Commun 301, 287-92 (2003).

35. Canzio, D., Larson, A. \& Narlikar, G.J. Mechanisms of functional promiscuity by HP1 proteins. Trends Cell Biol (2014).

36. Sridharan, R. et al. Proteomic and genomic approaches reveal critical functions of H3K9 methylation and heterochromatin protein-1gamma in reprogramming to pluripotency. Nat Cell Biol (2013).

37. Smallwood, A. et al. CBX3 regulates efficient RNA processing genome-wide. Genome Res 22, 1426-36 (2012).

38. Smallwood, A., Black, J.C., Tanese, N., Pradhan, S. \& Carey, M. HP1mediated silencing targets Pol II coactivator complexes. Nat Struct Mol Biol 15, 318-20 (2008).

39. Vakoc, C.R., Mandat, S.A., Olenchock, B.A. \& Blobel, G.A. Histone H3 lysine 9 methylation and HP1gamma are associated with transcription elongation through mammalian chromatin. Mol Cell 19, 381-91 (2005).

40. Liu, L.P., Ni, J.Q., Shi, Y.D., Oakeley, E.J. \& Sun, F.L. Sex-specific role of Drosophila melanogaster HP1 in regulating chromatin structure and gene transcription. Nat Genet 37, 1361-6 (2005).

41. Aydin, E. et al. A hypomorphic Cbx3 allele causes prenatal growth restriction and perinatal energy homeostasis defects. J Biosci 40, 325-38 (2015).

42. Naruse, C., Fukusumi, Y., Kakiuchi, D. \& Asano, M. A novel gene trapping for identifying genes expressed under the control of specific transcription factors. Biochem Biophys Res Commun 361, 109-15 (2007). 
43. Takada, Y. et al. HP1gamma links histone methylation marks to meiotic synapsis in mice. Development 138, 4207-17 (2011).

44. Wijchers, P.J. et al. Sexual dimorphism in mammalian autosomal gene regulation is determined not only by Sry but by sex chromosome complement as well. Dev Cell 19, 477-84 (2010).

45. Klein, S.L. \& Flanagan, K.L. Sex differences in immune responses. Nat Rev Immunol 16, 626-38 (2016).

46. Varlamov, O., Bethea, C.L. \& Roberts, C.T., Jr. Sex-specific differences in lipid and glucose metabolism. Front Endocrinol (Lausanne) 5, 241 (2014).

47. Blaak, E. Sex differences in the control of glucose homeostasis. Curr Opin Clin Nutr Metab Care 11, 500-4 (2008).

48. Clocchiatti, A., Cora, E., Zhang, Y. \& Dotto, G.P. Sexual dimorphism in cancer. Nat Rev Cancer 16, 330-9 (2016).

49. Mateescu, B., Bourachot, B., Rachez, C., Ogryzko, V. \& Muchardt, C. Regulation of an inducible promoter by an HP1beta-HP1gamma switch. EMBO Rep 9, 267-72 (2008).

50. Kim, H., Heo, K., Choi, J., Kim, K. \& An, W. Histone variant H3.3 stimulates HSP70 transcription through cooperation with HP1gamma. Nucleic Acids Res 39, 8329-41 (2011).

51. Gondos, B. Development and differentiation of the testis and male reproductive tract, (Raven Press, New York., 1980).

52. Conforto, T.L. \& Waxman, D.J. Sex-specific mouse liver gene expression: genome-wide analysis of developmental changes from pre-pubertal period to young adulthood. Biol Sex Differ 3, 9 (2012).

53. Wijchers, P.J. \& Festenstein, R.J. Epigenetic regulation of autosomal gene expression by sex chromosomes. Trends Genet 27, 132-40 (2011).

54. Goodfellow, P.N. \& Lovell-Badge, R. SRY and sex determination in mammals. Annu Rev Genet 27, 71-92 (1993).

55. Oh, H.J., Li, Y. \& Lau, Y.F. Sry associates with the heterochromatin protein 1 complex by interacting with a KRAB domain protein. Biol Reprod 72, 407-15 (2005).

56. Walport, L.J. et al. Human UTY(KDM6C) is a male-specific N-methyl lysyl demethylase. J Biol Chem 289, 18302-13 (2014).

57. Pedersen, M.T. \& Helin, K. Histone demethylases in development and disease. Trends Cell Biol 20, 662-71 (2010).

58. Heard, E. \& Disteche, C.M. Dosage compensation in mammals: fine-tuning the expression of the X chromosome. Genes Dev 20, 1848-67 (2006).

59. Yang, F., Babak, T., Shendure, J. \& Disteche, C.M. Global survey of escape 
from X inactivation by RNA-sequencing in mouse. Genome Res 20, 614-22 (2010).

60. Andreeva, A.V. \& Kutuzov, M.A. PPEF/PP7 protein Ser/Thr phosphatases. Cell Mol Life Sci 66, 3103-10 (2009).

61. Goff, L.A. et al. Spatiotemporal expression and transcriptional perturbations by long noncoding RNAs in the mouse brain. Proc Natl Acad Sci U S A 112, 6855-62 (2015).

62. Heard, E. et al. Methylation of histone H3 at Lys-9 is an early mark on the $\mathrm{X}$ chromosome during X inactivation. Cell 107, 727-38 (2001).

63. Chadwick, B.P. \& Willard, H.F. Chromatin of the Barr body: histone and nonhistone proteins associated with or excluded from the inactive $\mathrm{X}$ chromosome. Hum Mol Genet 12, 2167-78 (2003).

\section{Acknowledgements}

This work was supported by the Medical Research Council (MRC) (grant number MR/J007943/1). P.P.L was supported by the Joint University of Hong Kong-Imperial College London Ph.D. program. We thank the Medical Research Council London Institute of Medical Sciences (MRC LMS) Genomics Facility for Illumina sequencing services. Thanks to Prof. Christian Speck and Prof. Petra Hajkova for critical review of the manuscript. 


\section{Figure 1}

a

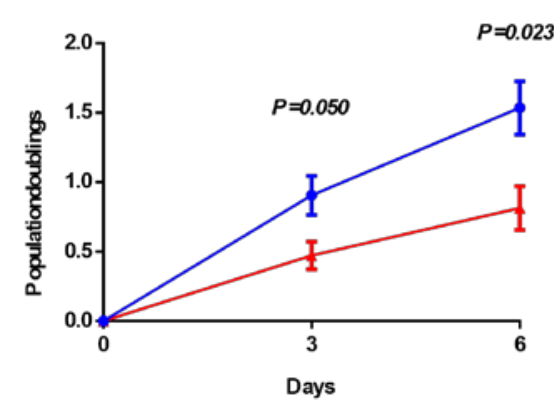

C

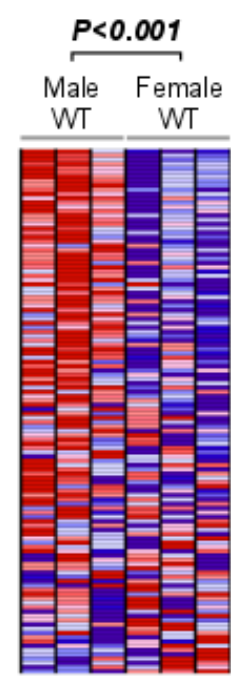

Low High b

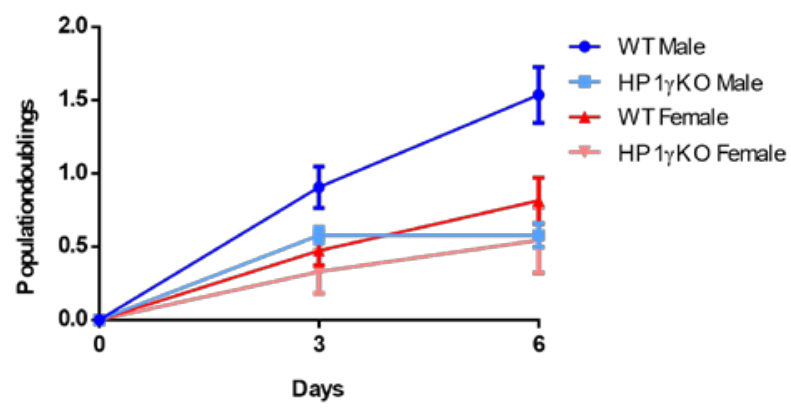

\begin{tabular}{|l|l|l|}
\hline P-value (T-test) & Day 3 & Day 6 \\
\hline WT male vs WT female & 0.050 & 0.023 \\
\hline WT male vs HP1yKO male & 0.144 & 0.007 \\
\hline WTfemale vs HP1yKOfemale & 0.366 & 0.285 \\
\hline WT male vs HP1yKOfemale & 0.041 & 0.015 \\
\hline WTfemale vs HP1yKO male & 0.422 & 0.260 \\
\hline
\end{tabular}

d
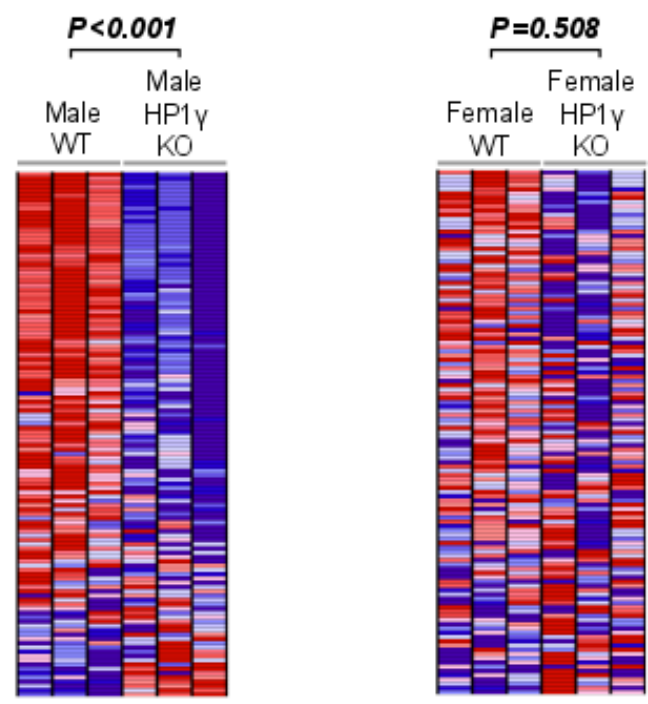

Low High

Figure 1. Sexually dimorphic proliferation rate of male and female E13.5 mouse embryonic fibroblasts (MEFs) is dependent on HP1 $\gamma$. a, b, Growth curve of male and female MEFs with or without HP1 $\gamma$ showing population doublings from day 0 to day 6. $n=8$ for WT male; $n=4$ for HP1 $\gamma$ KO male; $n=5$ for WT female; $n=3$ for HP $1 \gamma$ KO female. Error bars: SEM. P-values for each time point were obtained by two-sided Student's t-test. c-e, Genes with functions in cell cycle are affected by sex and HP1 $\gamma$ deletion. Gene set enrichment analysis (GSEA) of the transcriptome of male and female MEFs with or without HP1 $\gamma$ on cell cycle gene set. Comparisons were made between WT males and WT females (c), WT and HP1 $\gamma$ KO males (d) and WT and HP1 $\gamma$ KO 
females (e). The full list of genes shown on Fig. 1c to e can be found in extended Fig. 1a to c. Results were obtained from three MEF lines of each genotype derived from individual embryos. The data analysed here were derived from RNA-seq experiments that were repeated three times for each genotype. 


\section{Figure 2}
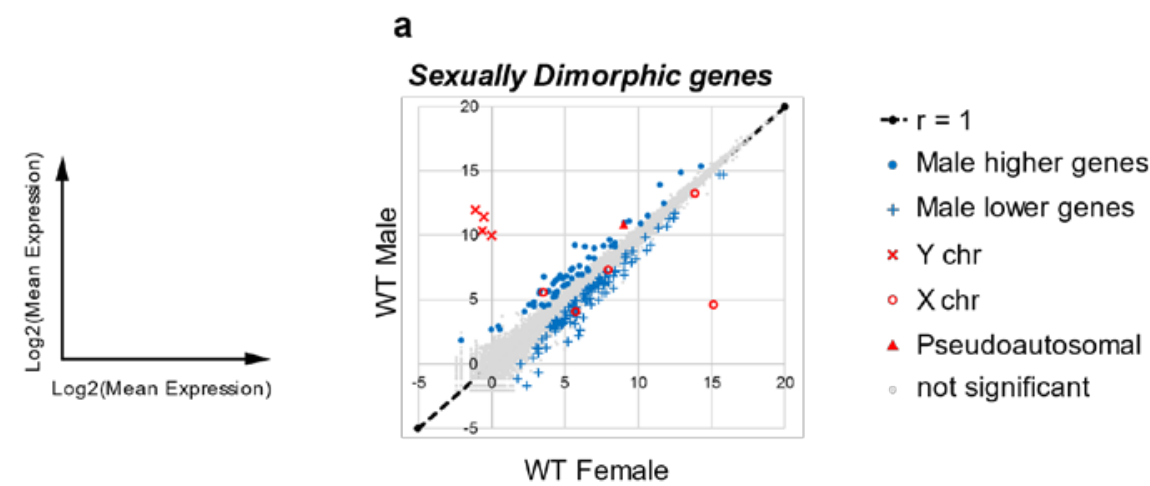

\section{b}

\section{Male-lower genes}
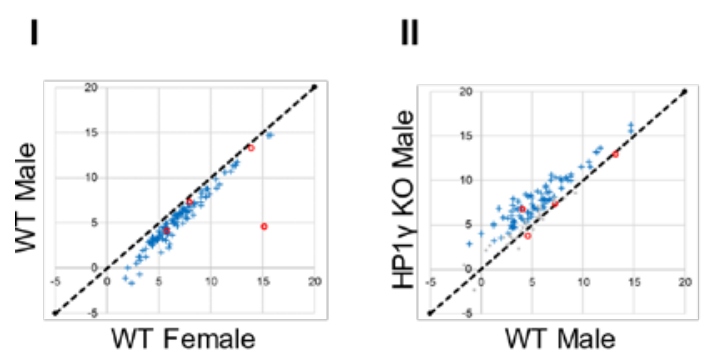

III

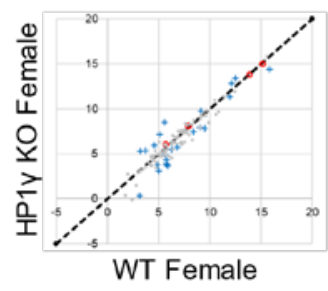

WT Female

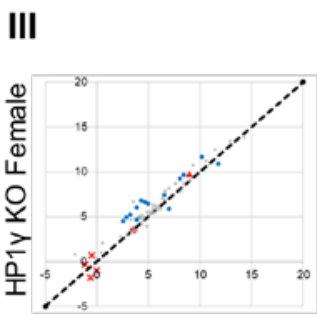

WT Female

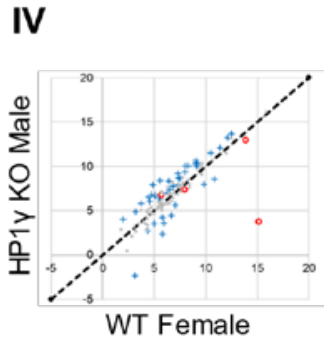

IV

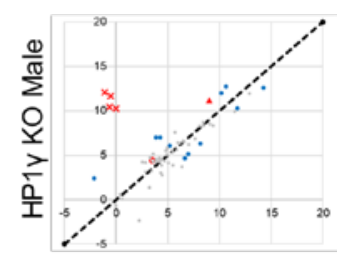

WT Female

Figure 2. HP1 $\gamma$ regulates sexually dimorphic autosomal genes. a, Density plot of differentially expressed genes comparing WT male and female MEFs. 176 genes (out of 23420 genes) display sexual dimorphic gene expression. b, Density plot of 114 'male-lower genes' which are repressed in WT males relative to WT females (b(I)). Upon HP1 $\gamma$ KO, most of the 'male-lower genes' were significantly upregulated in male (82 genes uperegulated; 0 gene downregulated; 32 genes not significantly changed) (b(II)). In female, most of these genes were not affected by HP1 $\gamma$ KO (7 genes uperegulated; 16 genes downregulated; 91 genes not significantly changed) (b(III)). Expression of 'male-lower genes' were also compared between HP1 $\gamma$ KO male and WT 
female MEFs (33 genes showed higher expression in males,10 genes showed higher expression in females and 67 genes not significantly different between two sexes exclusive of sex chromosome genes) ((b(IV)). c, Density plot of 62 'male-higher genes' which are more highly expressed in WT males relative to WT females (c(I)). In males, most of the 62 'male-higher genes' were downregulated in absence of HP1 $\gamma$ (3 genes uperegulated; 35 genes downregulated; 24 genes not significantly changed) (c(II)). A minimal effect was observed in females upon HP1 $\gamma$ KO (13 genes uperegulated; 2 genes downregulated; 47 genes not significantly changed) (c(III)). Expression of 'malehigher genes' were also compared between HP1 $\gamma$ KO male and WT female MEFs (6 genes showed higher expression in males; 5 genes showed higher expression in females; 45 genes not significantly different between two sexes exclusive of sex chromosome genes) (c(IV)). Results were obtained from three MEF lines of each genotype derived from individual embryos. Therefore, the data analysed here were derived from RNAseq experiments that were repeated three times for each genotype. Genes showing $\geq 1$.5fold difference with P-value $<0.05$ between conditions compared were defined to be statistical significantly changed. 


\section{Figure 3}

a

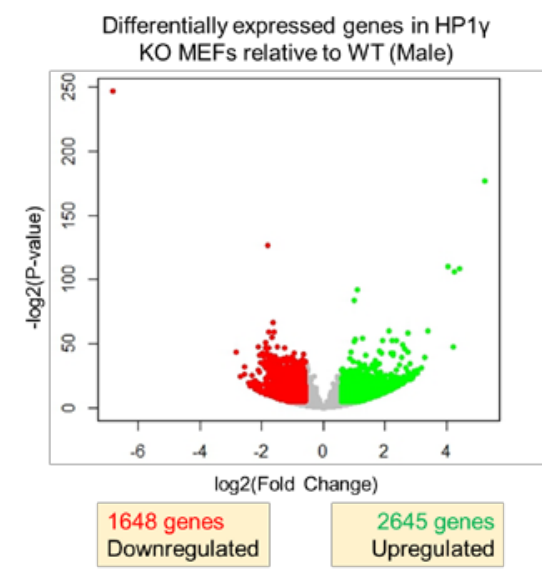

b Differentially expressed genes in HP1y KO

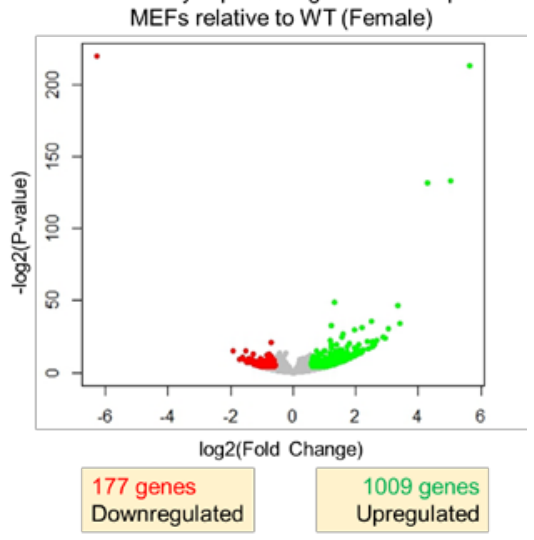

C

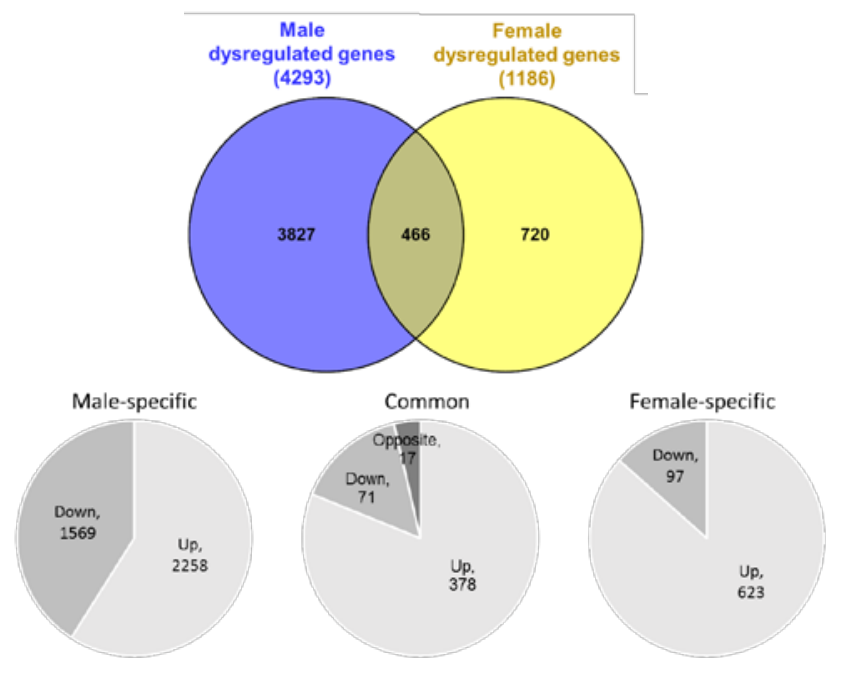

Figure 3. HP1 $\gamma$-deficient MEFs display a male-biased transcriptional defect Differentially expressed genes in male (a) and female (b) MEFs in response to knockout of HP1 $\gamma$ was revealed by RNA-seq. Results were obtained from three MEF lines of each genotype derived from individual embryos. (Fold change $\geq 1.5, \mathrm{P}<0.05$ ). $\mathbf{c}$, Genes differentially expressed in male and female HP1 $\gamma$-deficient MEFs and the number of genes up- or down-regulated or differentially expressed in opposite directions in each sex. The data analysed here were derived from RNA-seq experiments that were repeated three times for each genotype. 


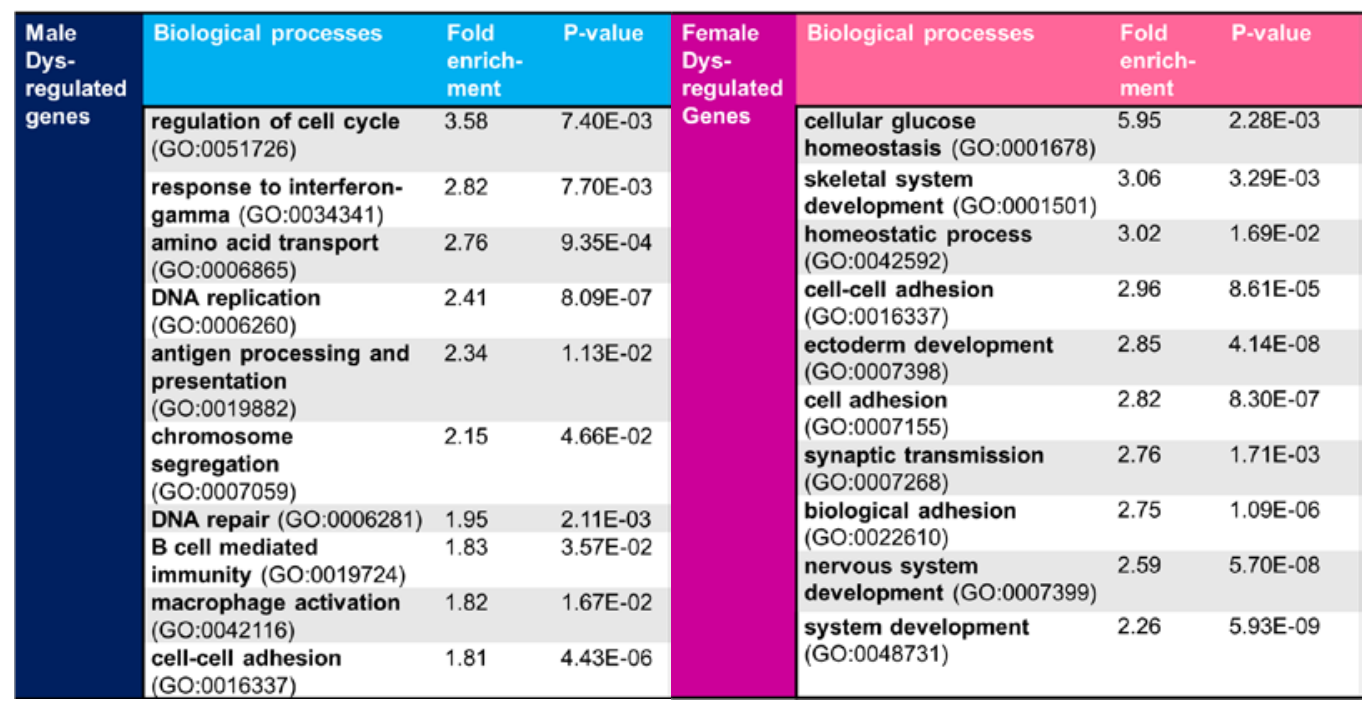

Figure 4. Sex-biased dysregulation of physiological functions upon HP1 $\gamma$ knockout. List of top 10 biological processes revealed to be associated with genes specifically dysregulated in males or females. 